\title{
Estrés y resiliencia en el contexto del COVID-19, en estudiantes de educación secundaria
}

\section{Stress and resilience in the context of COVID-19, in high school students}

\author{
DORIS CASTRO HUAMANI ${ }^{1}$ \\ dofranris@gmail.com \\ https://orcid.org/0000-0002-2584-0503 \\ Institución Educativa Secundaria Julio Alberto \\ Ponce Antúnez de Mayolo, Sicuani, Perú \\ FrankLin TAIPE FLOREZ ${ }^{3}$ \\ ftaipef@unsa.edu.pe \\ https://orcid.org/0000-0002-3639-3892
}

\author{
VILMA CASTRO HuAMANI ${ }^{2}$ \\ virmacastro1@gmail.com \\ https://orcid.org/0000-0003-1516-8351 \\ Institución Educativa Secundaria Inmaculada \\ Concepción, Sicuani, Perú \\ Sonia Castro huamani ${ }^{4}$ \\ jesucadj@gmail.com \\ https://orcid.org/0000-0001-9806-4261
}

Unidad de Gestión Educativa Local de Canas, Perú

\begin{abstract}
RESUMEN:
El estudio de las consecuencias sanitarias en el sector educación es significativo. En ese sentido, el propósito del trabajo de investigación es determinar la correlación de la confianza en sí mismo y el sentirse bien solo, como resiliencia y el estrés académico en alumnos de quinto grado de educación secundaria en aulas piloto de un colegio público en el contexto del COVID-19. Este trabajo se realizó con un enfoque cuantitativo, de tipo básico y metodología descriptiva, correlacional, donde se encuestaron virtualmente a 52 estudiantes escogidos por "criterio-juicio" (Tamayo, 2001), usando el Rango de resiliencia (Wagnild y Youbg, 1993) y la adecuación del cuestionario de estrés académico SISCO SV al entorno del COVID-19 (Alania et al., 2020). Existe relación significativa negativa media para la confianza en sí mismo y en forma considerable para sentirse bien solo, con el estrés $(r=-.656$ y $r=-.717)$ respectivamente $(p<0.01)$, concordante con otros estudios. Sosteniendo que estudiantes de aulas piloto que cuentan con apoyo material y familiar tienen mayores capacidades de resiliencia desde su "confianza en sí mismo" y el "sentirse bien solo". Por lo tanto, sufren menor estrés académico en el contexto de la educación virtual por el COVID-19, por lo cual el trabajo debe estar dirigido a empoderar las capacidades de resiliencia desde la familia y el colegio.
\end{abstract}

\section{ABSTRACT:}

It is important to study the consequences of the current crisis in education, the purpose is to determine the correlation of self-confidence and feeling good alone, such as resilience and academic stress in fifth graders of secondary education in pilot classrooms of a public school in the context of COVID-19. This work was carried out with a quantitative approach, basic type and descriptive, correlational methodology, where virtuaIly 52 students chosen by "criterion-judgment" were surveyed (Tamayo, 2001), using the Resilience Range (Wagnild \& Young, 1993) and the adaptation of the SISCO SV academic stress questionnaire to the COVID-19 environment, by Alania et al. (2020). There is a mean negative significant relationship for self-confidence and in a considerable way to feel good alone, with stress $(r=-.656$ and $r=-.717)$ respectively $(p<0.01)$, consistent with other studies. Holding that students from pilot classrooms that have material and family support have greater resilience capacities from their "self-confidence" and "feeling good alone"; therefore, they suffer less academic stress in the context of virtual education due to COVID-19, so the work must be aimed at empowering resilience capacities from the family and school.

\footnotetext{
(C) Los autores. Este artículo es publicado por la Revista peruana de investigación e innovación educativa de la Facultad de Educación, Universidad Nacional Mayor de San Marcos. Este es un artículo de acceso abierto, distribuido bajo los términos de la licencia Creative Commons Atribución 4.0 Internacional (CC BY 4.0) [https://creativecommons.org/licenses/by/4.0/deed.es] que permite el uso, distribución y reproducción en cualquier medio, siempre que la obra original sea debidamente citada de su fuente original.
} 


\section{Palabras Clave:}

Contexto covid-19; estrés académico; confianza en sí mismo; sentirse bien solo; resiliencia.

\section{KEYWORDS:}

Covid-19 context; academic stress; self-confidence; feeling good alone; resilience.

Recibido: 15/09/2021 - Aceptado: 10/10/2021 - Publicado: 23/12/2021

\section{Introducción}

La crisis por el COVID-19 está generando problemas de estrés y de salud mental en todos los sectores de la población, afectando a los sectores productivo, económico, laboral, social, deportivo y cultural (Pérez et al., 2020), siendo más notorio en el sector educación. Dicha situación afecta a los maestros y estudiantes (Gonzáles, 2020; Raymundo, 2021), el cierre de los colegios, el aislamiento y la educación virtual, han provocado en los estudiantes estrés (Vivanco et al., 2021). Por lo que se hace urgente atender esta problemática con estrategias de afrontamiento, de lo contrario estos pueden agravarse al no ser atendidos a nivel mundial (Kang et al., 2020).

En el Perú y en el mundo el problema del estrés traerá mayores consecuencias en un futuro próximo (Palomino y Huarcaya, 2020; Rodriguez, 2021), por lo que, resulta crucial tener que afrontarla desde factores externos y mucho más desde las propias capacidades humanas de resiliencia como (Serrano y Sanz, 2019) no solo por los profesionales de primera línea (Solano, 2020), en especial por los docentes y estudiantes debido a la educación virtual (Roman et al., 2020), para sobreponerse a la crisis del contexto.

La plataforma Aprendo en casa del Gobierno del Perú, como soporte para la educación virtual tiene, a pesar de sus oportunidades, dificultades que han generado un estrés en los estudiantes especialmente adolescentes (Jacobo, 2020; Linares, 2021; Mamani, 2020;), por lo que, es importante que la familia, la escuela y el sistema puedan proporcionar un soporte que empodere las capacidades de resiliencia, debido a que son muy necesarias para confrontar la situación estresante (León, 2020).

El fenómeno del estrés aparece de manera gradual, la persona lo asume progresivamente (Melgosa,1995; Naranjo, 2009); entonces, las sesiones virtuales en el contexto del COVID-19, pueden generar estrés como respuesta a la demanda del medio exterior tanto de manera física o emocional (Díaz, 2011), en particular el estrés académico. Caldera et al. (2007) asumen que todo un conjunto de factores internos y externos, los cuales afectan a profesores y estudiantes, conllevan a generar cuadros de estrés en el contexto educativo.

El estrés al interior de las instituciones educativas, puede expresarse como un rasgo perceptivo y subjetivo de las distintas situaciones, acciones, condiciones que experimenta en el aula y fuera de ella y que puede llegar a perjudicar el normal desarrollo estudiantil, reduciendo la concentración y calidad del aprendizaje (Asenjo et al., 2021; Barraza, 2008; Caqueo et al., 2020).

La resiliencia lo que conlleva a saltar o evitar (Kotliarenco et al., 1997), es una capacidad humana de afrontar y sobrellevar situaciones dificultosas (Uriarte, 2005; Velezmoro, 2018), como respuestas y acciones asertivas e inteligentes en situaciones riesgosas, siendo en un inicio reacciones innatas y luego asimiladas y elaboradas con la propia experiencia (Wagnild y Young, 1993). Además, Novella (2002), menciona que la resiliencia es posibilidad que se tiene de adecuar nuestro organismo a los cuadros de estrés que podríamos enfrentar.

Para Luthar et al. (2000), la resiliencia es una adaptación frente a experiencias negativas. Esta cualidad es primordial en estos tiempos críticos, según Imbert (2020) y Lozano (2020), se está poniendo de manifiesto en el contexto de pandemia actual (Castagnola et al., 2021). 
La resiliencia posee muchas dimensiones (Novella, 2002; Wagnild y Young, 1993), para el presente estudio se tendrán en cuenta: "confianza en sí mismo", como las habilidades, capacidades y cualidades internas con fuerza. Para M. Pérez et al. (2020), la persona que presenta signos de autoconfianza, actúa con capacidad y autonomía frente a las adversidades de la vida. Según Wagnild y Young (1993) y Novella (2002) consideran a la actuación individual del sujeto como el inicio de la posibilidad que se tiene para superar las dificultades. Dentro de todo un conjunto de características que se relacionan podríamos mencionar al optimismo frente a la vida, el establecer metas personales, la persistencia, entre otros (López y Calvetel, 2016; Reyes, et al., 2017).

El estrés y resiliencia con sus distintas dimensiones tienen una complementariedad de acción y es común el estudio de ambas variables para ver su relación que no siempre es la misma y que también tiene influencia del grupo humano en el estudio, el contexto y otros factores (Caldera et al., 2007; Kim, 2019; Salvatierra, 2019), y muy en especial interés tiene en la situación de pandemia y aislamiento (Correa y Mendoza, 2020; Gonzáles, 2020; Verastegui, 2020).

El contexto del COVID-19 está delimitado por lo sanitario, económico, político por mencionar algunos contextos macros y que tuvieron su epicentro en la región de Wuhan (China), a finales del año 2019 (Huarcaya, 2020; Velaban y Meyer, 2020), además de generar ansiedad e incertidumbre (Oltra y Boso, 2020), especialmente, en los estudiantes de todos los niveles (Caqueo et al., 2020).

El estudio pretende indagar sobre las relaciones que pudieran existir entre las dimensiones de la resiliencia, como capacidades de los estudiantes frente al estrés académico por la educación virtual en el contexto del COVID-19. Por lo que, resulta necesario e importante conocer estas relaciones porque servirían de información a los docentes, padres de familia y administradores educativos, para planificar políticas y estrategias de intervención que ayuden a los estudiantes y así puedan lograr las competencias que contempla el Currículo Nacional de Educación Básica (CNEB, 2017). Asimismo, el propósito de la investigación fue hallar la correlación entre las dimensiones: "confianza en sí mismo" y "sentirse bien solo", de la resiliencia y el estrés académico, en alumnos del quinto grado de educación secundaria, de las aulas piloto, en el entorno del COVID-19.

El trabajo se enmarca en los aspectos teóricos del estrés tipo académico, así como la resiliencia, y en como ambos conceptos pueden tener relaciones. Román y Hernández (2011) refieren que el estrés académico se manifiesta como sensaciones de inconformidad a la demanda educativa que generan problemas de aprendizaje y la resiliencia (Novella, 2002), como actitudes y aptitudes para enfrentar y resolver esas inconformidades, hasta sobreponerse positivamente (Barraza y Silerio, 2007; Barraza y Oscar, 2010; Becońa, 2006; Canazas y Diaz, 2019).

El estudio de Verastegui (2020) en el contexto educativo superior investigar acerca del estrés y la resiliencia. Para ello empleo el enfoque cuantitativo y con diseño correlacional bivariado. Se empleó el instrumento SISCO para analizar la primera variable; en el caso la resiliencia, esta fue medida a través de la propuesta de Wagnild y Young. Se encontró evidencia de correlación negativa moderada $(r=-.616$ y p=.000), del estrés y la resiliencia genérica y estrés con la dimensión "confianza en sí mismo", correlación inversa débil ( $\mathrm{r}=-.260$ y $\mathrm{p}=.000$ ). Concluyendo que, la reducción del estrés académico presente se debe al incremento de la resiliencia que se ofrece, por lo cual deben impulsarse competencias blandas y aptitudes resilientes.

Para Salvatierra (2019), en su investigación sobre resiliencia y estrés académico, el propósito fue determinar la relación de la resiliencia, en el estrés académico para alumnos de un conservatorio de música. Dicho trabajo fue planteado con diseño correlacional y los instrumentos de rango de resiliencia de Wagnild y Young (1993), adecuado por Nunja (2016) y el inventario SISCO del estrés tipo académico (Barraza, 2008) adecuado por Anjicama (2017). Encontrando una correlación inversa negativa ( $\mathrm{r}=-.22$ y p=.004) en lo general. Concluyendo que a menor estrés académico mayor resiliencia, con respecto a la dimensión "sentirse bien solo" ( $\mathrm{r}=-.27$ y 
$\mathrm{p}<0.05)$, a "confianza" ( $\mathrm{r}=-.39$ y $\mathrm{p}<0.05)$, sobre el estrés, evidenciando que a mejor actitud a sentirse bien solo y con autoconfianza, menor es la carga del estrés académico.

Leon et al. (2019), al relacionar el estrés, autoeficacia y rendimiento académico, con la resiliencia reporta una investigación en adultos emergentes de 18 a 25 ańos. Esta fue un estudio correlacional y empleo instrumentos como la escala de autoeficacia en conductas académicas y la encuesta de resiliencia. La correlación de Pearson para el índice global de estrés y resiliencia fue $\mathrm{r}=-.193$ y $\mathrm{p}<0.01$. El estudio concluye que, al aumentar el rango del estrés académico, la resiliencia se reduce, donde el conjunto calificado como de baja resiliencia posee un rendimiento académico con ligera desventaja con respecto al conjunto de calificación con mayor resiliencia.

Mientras que el estudio de Maldonado y Paucar (2021), sobre estrés y resiliencia en universitarios cuyo propósito fue determinar la relación entre a mbas variables; empleado una metodología cuantitativa y relacional de tipo básico, aplicándose el cuestionario SISCO. El estadístico estableció que no existe relación para las variables $(\mathrm{p}=0.915>0.05)$, refiriendo que se debe reducir el estrés académico de manera grupal e individual con talleres de habilidades sociales y relajación.

El hallazgo de García et al. (2021), en su estudio de la resiliencia y estrés percibidos por los estudiantes de obstetricia tuvo como meta fue establecer la relación de la resiliencia con el estrés, de una universidad pública de Lima. Se empleo un estudio analítico y transversal, además de una escala de resiliencia y el rango de percepción global del estrés, estableciendo una correlación inversa moderada para las variables (rho=-0,496 y $\mathrm{p}=<0.001$ ), el estrés con la dimensión "competencia personal" (rho $=-0,53$ y $\mathrm{p}=<0.001)$, y con "aceptación de sí mismo" (rho $=-0,387$ y $\mathrm{p}=<0.001$ ). Se refirió que la resiliencia se comporta conforme a una relación inversa considerable frente al estrés siendo preciso reducir esta ocurrencia en la etapa universitaria.

La investigación de Rojas (2016) tuvo como propósito determinar investigar la resiliencia y afrontamiento al estrés en estudiantes de educación básica. El trabajo tuvo un diseño descriptivo-correlacional, se empleó en el cuestionario de estrés (CAE) y el rango de resiliencia (ERA), encontrándose que no existe correlación estadística entre resiliencia y afrontamiento de estrés $(\mathrm{r}=0.08$ y $\mathrm{p}<0.005)$. Adicionalmente, también es nula entre la dimensión de independencia, iniciativa de la resiliencia y el afrontamiento de estrés $(\mathrm{r}=0.07$ y r=0.06) respectivamente. Refiriendo de esta manera la relación positiva entre la dimensión creatividad de la resiliencia y del afrontamiento del estrés ( $\mathrm{r}=0.12$ y $\mathrm{p}<0.005)$, y que los factores de situaciones adversas cotidianas pueden servir como estímulos para sobresalir y superar las circunstancias.

\section{Método}

La investigación fue de tipo básico, porque se originó en un marco teórico y con el fin de incrementar un conocimiento teórico sobre la intersección entre resiliencia y estrés académico, sin contrastación práctica (Muntane, 2010). Tuvo un carácter descriptivo correlacional, porque se describió la correlación de la resiliencia y estrés académico en el contexto del COVID-19 (Hernández et al., 2014). El diseńo fue no experimental, transversal, porque no se manipularon variables y los instrumentos se aplicaron en un solo corte (Hernández y Velazco, 2000). La técnica utilizada fue la encuesta virtual, por el aislamiento social con la herramienta Google Forms, como en los estudios en el contexto de aislamiento por la crisis sanitaria Cañete et al. (2021). Por la naturaleza de la encuesta se tomaron las nociones de Hernandez et al. (2014). Además, la encuesta usó enunciados en forma de preguntas estructuradas, y que reunieron información para el tratamiento estadístico.

Se utilizaron dos instrumentos, para la variable del estrés académico en el entorno del COVID-19, el "Inventario de estrés académico SISCO SV, adaptado al entorno de la crisis por la COVID-19" de Alania et al. (2020) con 45 ítems y tres dimensiones, estresores, síntomas, afrontamiento; mientras que para la variable de resiliencia se empleó el instrumento cuestionario: "Escala de Resiliencia" (Wagnild y Young, 1993) 
adecuado por (Novella, 2002) con 25 ítems y con los aspectos de ecuanimidad, perseverancia, confianza en sí mismo, satisfacción personal y sentirse bien solo.

La muestra de estudio fue de 52 estudiantes matriculados en el quinto año de secundaria, en las secciones piloto, del $5^{\circ}$ grado "D" y $5^{\circ}$ grado "E" de una institución educativa pública. El muestreo fue del tipo no probabilístico, por el interés de la investigación, ya que, fue por conveniencia según "criterio-juicio", porque se tomaron los elementos de acuerdo con el criterio y juicio del investigador, como sugiere Tamayo (2001). Los criterios de selección fueron, estudiantes de aulas "piloto", estudiantes con desempeño académico favorable, apoyo familiar, y condiciones medias favorables en rendimiento académico (Treviño et al., 2016).

Los datos fueron procesados con el software SPSS-24, para el análisis inferencial se utilizó el estadístico rho de Spearman, con pruebas a un nivel de confianza del 95\% ( $\mathrm{p}<0.05)$. Los instrumentos se aplicaron con el conocimiento y autorización de los estudiantes, así como de la dirección del colegio, además en el trabajo se expone pleno respeto al aporte de producción intelectual de los autores citados.

\section{Resultados}

Los resultados del nivel de estrés académico y de la resiliencia en el entorno del COVID-19, se aprecian en la tabla 1 y las frecuencias de las dimensiones de: confianza sí mismo y sentirse bien solo de la resiliencia, en la tabla 2 .

Tabla 1

Frecuencias: Estrés Académico y Resiliencia

\begin{tabular}{lccccc}
\hline \multicolumn{2}{c}{ Estrés académico en el contexto: COVID-19 } & \multicolumn{3}{c}{ Resiliencia en el contexto: COVID-19 } \\
\multicolumn{1}{c}{ Nivel } & Frecuencia & Porcentaje & Nivel & Frecuencia & Porcentaje \\
Leve & 32 & $61.50 \%$ & Bajo & 00 & $00.00 \%$ \\
Moderado & 14 & $26.90 \%$ & Medio & 07 & $17.30 \%$ \\
Grave & 06 & $11.60 \%$ & Alto & 45 & $82.70 \%$ \\
\hline Total & 52 & & Total & 52 & \\
\hline
\end{tabular}

Nota. Elaborado con datos obtenidos.

La encuesta reporta que la mayoría de los alumnos tuvieron un rango de estrés leve y un bajo porcentaje en el nivel grave; así como un nivel alto de resiliencia y ningún estudiante reporta un nivel bajo de resiliencia.

Tabla 2

Frecuencias de "Confianza en sí Mismo" y "Sentirse Bien Solo"

\begin{tabular}{lcccc}
\hline & \multicolumn{2}{c}{ Confianza en sí mismo } & \multicolumn{2}{c}{ Sentirse bien solo } \\
\multicolumn{1}{c}{ Nivel } & Frecuencia & Porcentaje & Frecuencia & Porcentaje \\
\hline Bajo & 00 & $00.0 \%$ & 01 & $01.9 \%$ \\
Medio & 07 & $13.5 \%$ & 09 & $17.3 \%$ \\
Alto & 45 & $86.5 \%$ & 42 & $80.8 \%$ \\
\hline Total & 52 & & 52 & \\
\hline
\end{tabular}

Nota. Elaborada en base a los resultados obtenidos.

Los estudiantes mostraron resiliencia para ambas dimensiones con niveles altos, siendo casi nulo un nivel bajo. El comportamiento de la correlación cruzada de estrés académico con la resiliencia se observa en la figura 1, mientras que la relación cruzada entre el estrés y las dimensiones: confianza en sí mismo y sentirse bien solo, de la resiliencia, se muestran en las figuras 2 y 3 respectivamente. 
Figura 1

Relación Cruzada: Estrés Académico con Resiliencia en el Entorno del COVID-19

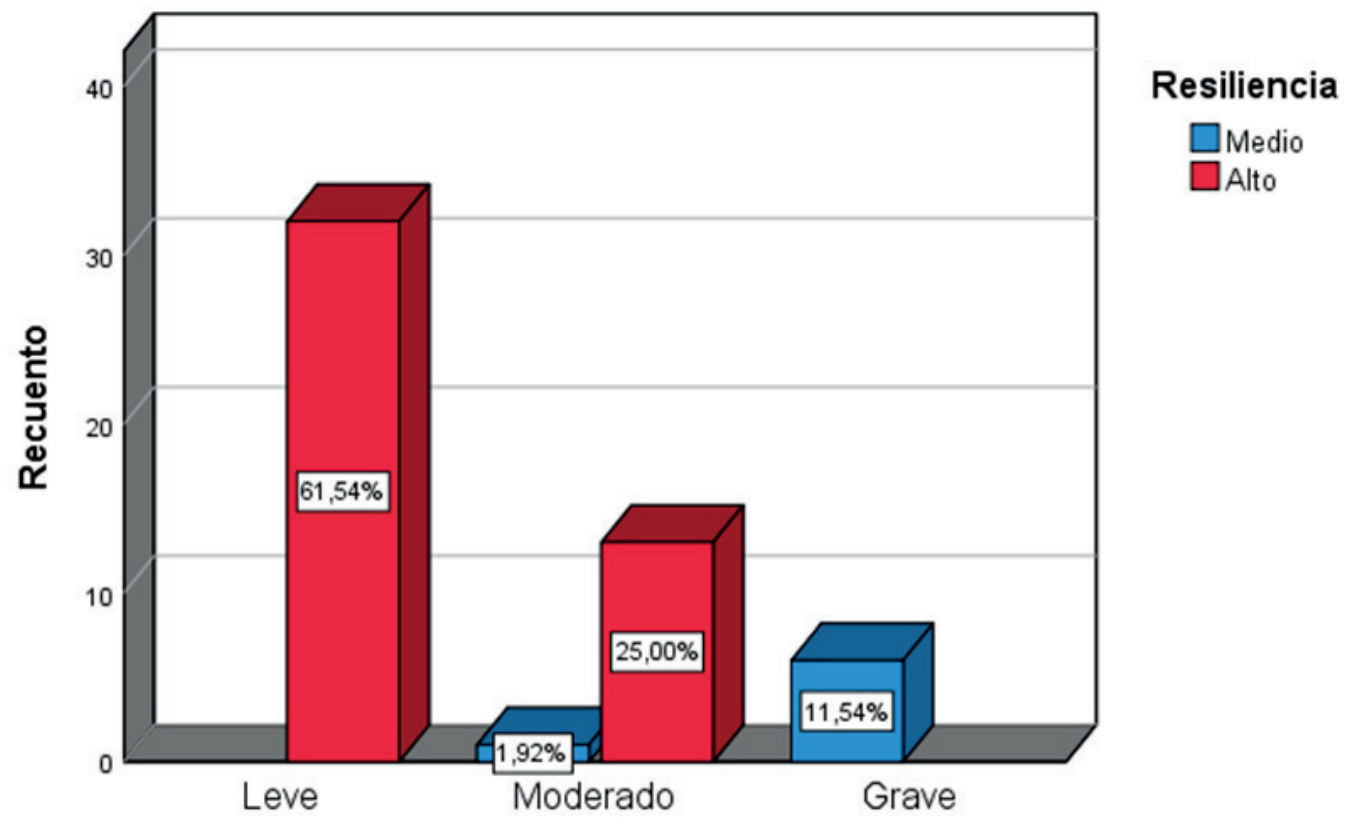

Estres en el contexto COVID-19.

Es notoria la correlación entre el nivel leve de estrés académico y la percepción de una resiliencia alta.

Figura 2

Relación Cruzada: Estrés Académico y la Confianza en sí Mismo de la Resiliencia

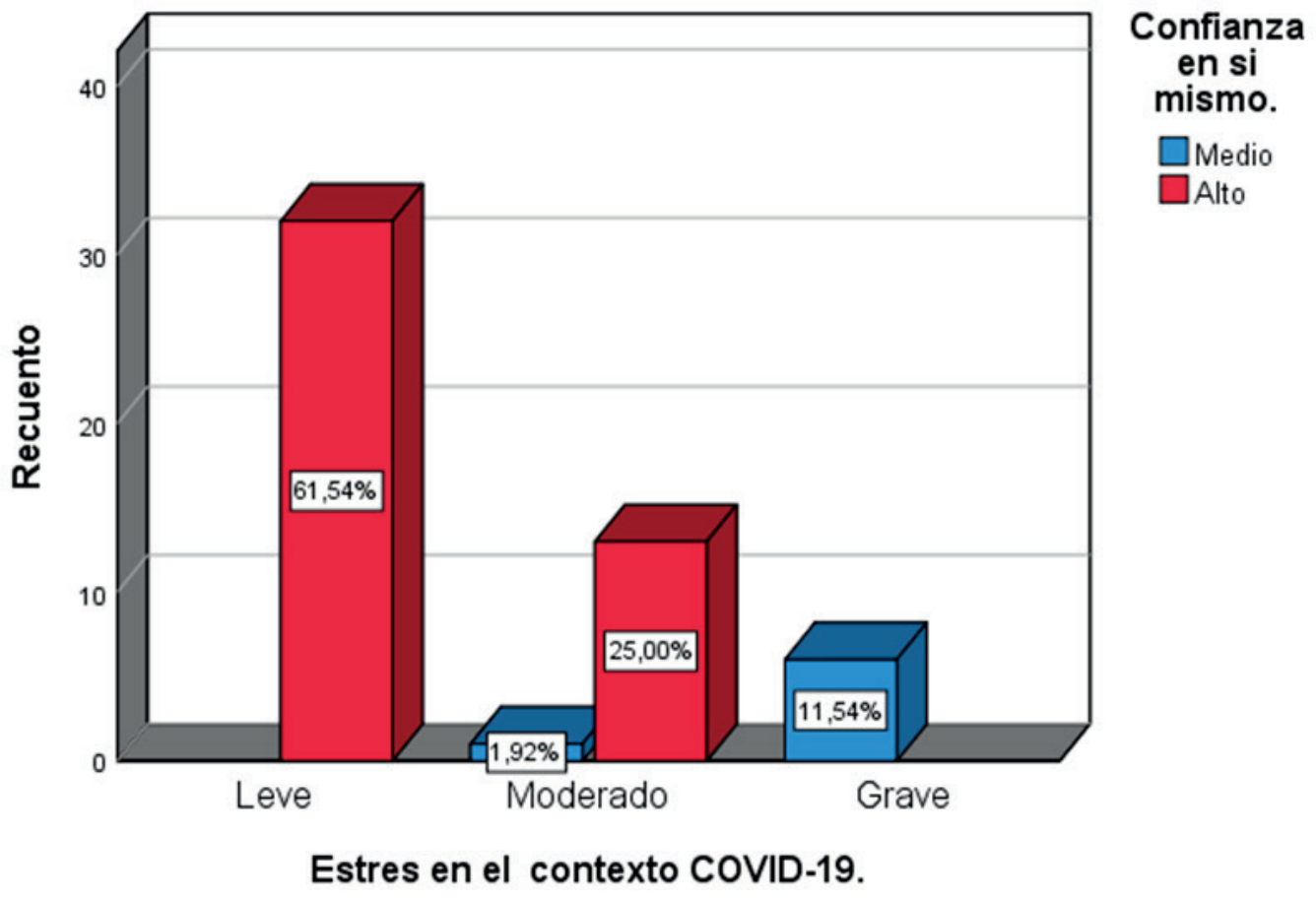

En el nivel leve de estrés académico, la percepción de un rango de confianza en sí mismo fue alto. 
Figura 3

Relación Cruzada del Estrés Académico y El Sentirse Bien Solo, de la Resiliencia

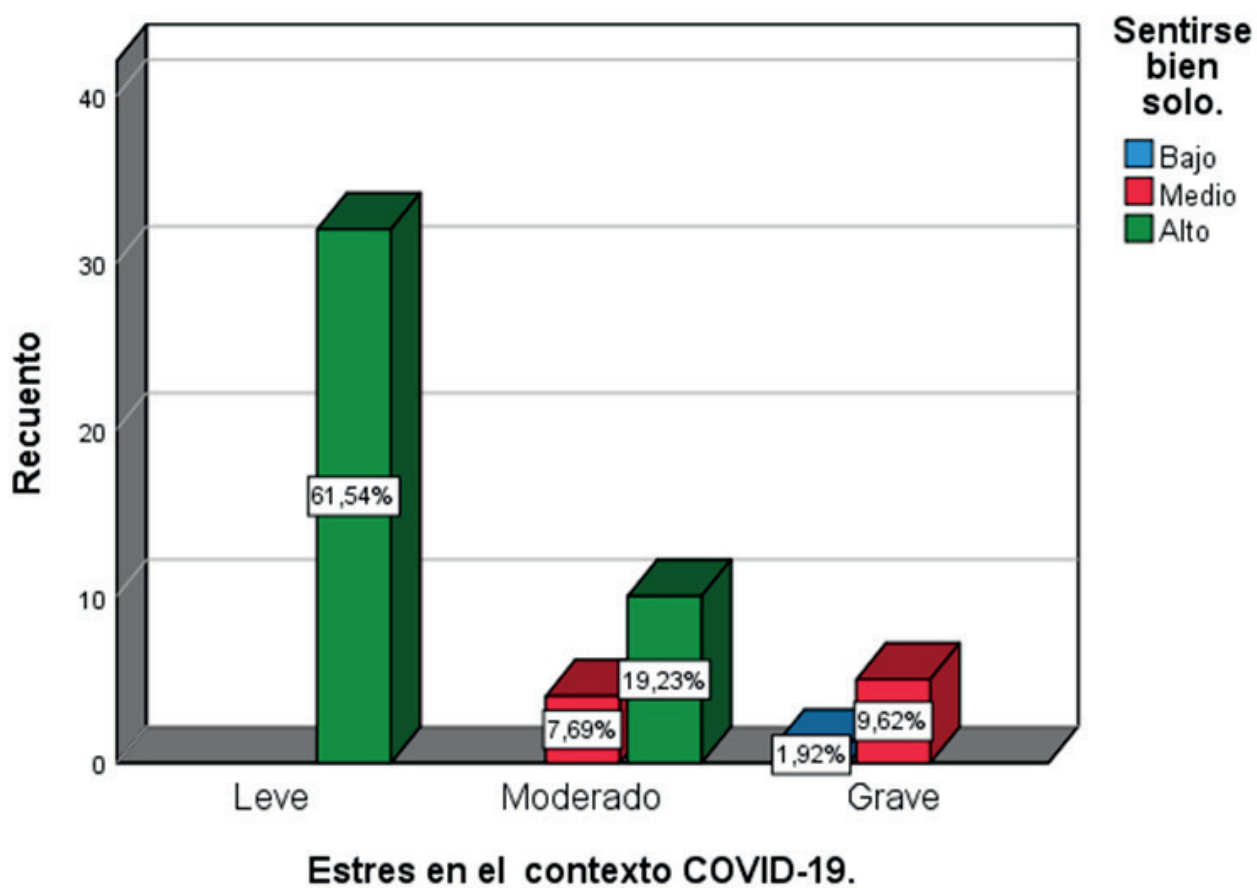

En el nivel leve de estrés, se percibió un nivel alto de resiliencia, con la dimensión de sentirse bien solo. Por el número de estudiantes en la muestra, se utilizó la prueba de Kolmogórov-Smirnov con ( $<<0.01)$. La muestra no proviene de una distribución normal, por lo cual se usó técnicas no paramétricas para la prueba de hipótesis y el estadístico ( $\rho=$ rho, Spearman), como refiere Tamayo (2001), la que se observa en la tabla 3.

Tabla 3

Coeficiente: Correlación Bilateral

\begin{tabular}{lc}
\hline Estrés académico en el contexto: COVID-19 & Coeficiente de correlación rho de Spearman \\
Resiliencia & $-.656^{* *}$ \\
Confianza en sí mismo & $-.656^{* *}$ \\
Sentirse bien solo & $-.717^{* *}$ \\
\hline Nota: Elaborada en base a resultados $(* *$ corresponde $p<0.01)$ &
\end{tabular}

Por observación de la tabla 3, se evidenció un grado de correlación entre el "Estrés en el contexto del COVID-19", con la "resiliencia", "confianza en sí mismo" y "sentirse bien solo", como relaciones negativas próximas a ser considerados, significando que, por ejemplo, a menor estrés académico mayor resiliencia.

\section{Discusión}

La investigación tuvo como meta, determinar la relación de la resiliencia con el estrés académico en el contexto del COVID-19, resultando una relación negativa próxima a la considerable $(r=-, 656$ y $\mathrm{p}<0.01)$ como refiere Martinez et al. (2009). Se puede apreciar en la figura 1, como en el nivel leve y moderado de estrés se percibe un nivel alto de resiliencia de (61.54\% y $25.0 \%)$ respectivamente, afirmando con evidencia estadística que los estudiantes de las aulas piloto del quinto grado de secundaria, presentan menor estrés académico porque mayor es su nivel de resiliencia. Este resultado es análogo al reporte de Berzosa (2016), que indica una correlación negativa de estresores y resiliencia $(\mathrm{r}=-.206$ y $\mathrm{p}<0.05)$ en estudiantes universitarios, también con Salvatierra (2019) en estudiantes jóvenes $(\mathrm{r}=-.22$ y p=0.004), con el estudio de Leon et al. (2019), con una relación inversa débil $(\mathrm{r}=-.193$ y $\mathrm{p}<0.01)$, en una época distinta a la crisis por la pandemia, discordante con el estudio de 
Morales (2017) reportando ( $\mathrm{r}=.182$ y p $<0.05)$ que concluye en una correlación positiva débil de la resiliencia y afrontamiento del estrés académico en alumnos universitarios, discordante también con los trabajos de Rojas (2016), Maldonado y Paucar (2021), que informa de no haber ninguna relación entre ambas variables.

Para estudios enmarcados en la crisis por la pandemia, se tiene concordancia con Verastegui (2020), con una correlación negativa moderada ( $\mathrm{r}=-.616$ y p=.000), al igual que García et al. (2021) (rho=-0,496 y $\mathrm{p}=<0.001)$; es decir, cuánto más estrés académico hay, menor es el nivel de resiliencia. En el estudio, el resultado de la relación puede ser sustentada por la naturaleza de la muestra, ya que, son adolescentes entre 15 y 16 ańos que muestran una fuerza ante la adversidad según Cahuana y Carazas (2018), una actitud de sobreponerse y ver el día a día, como una nueva historia como indica Moran (2018) y Gonzáles (2019). Pero, la cualidad más importante para esta relación, se atribuye a su condición escolar en un aula piloto, donde los alumnos tienen la ayuda y monitoreo de sus padres y familias, un rendimiento académico favorable a muy favorable, predisposición al trabajo colaborativo y con autonomía (CNEB, 2017), estas características son paralelas a las aptitudes resilientes que señala Becoña (2006), como lo refieren también los estudios de Mendez (2020) y Cabrera et al. (2019).

Para la meta en determinar la correlación entre el "estrés académico en el contexto del COVID-19" y la "Confianza en sí mismo" en los estudiantes, esta fue superior a una correlación negativa media ( $\mathrm{r}=-.656$ y p $<0.01)$, con percepción alta de resiliencia de $(65.6 \%$ y $25.0 \%)$ para el nivel de estrés leve y moderado respectivamente, como muestra la figura 2. Concluyéndose con evidencia estadística que, a mayor nivel de resiliencia con su dimensión "confianza en sí mismo", se tiene un menor rango de estrés académico en el entorno del COVID-19, lo cual, es concordante con Verastegui (2020) y su relación ( $r=-.260$ y p=.000), con Salvatierra (2019), que reporta una correlación superior a una negativa débil ( $r=-.27$ y $\mathrm{p}<0.05)$, haciendo el paralelo entre las dimensiones: confianza en sí mismo y competencia, se coincide con García et al. (2021) para estudiantes universitarios en el contexto del COVID-19; pero, es contrario al estudio de Rojas (2016) que, en estudiantes de secundaria no encontró relación significativa $(\mathrm{r}=0.07)$.

La "confianza en sí mismo" como una capacidad presente en los estudiantes resulta muy beneficiosa para afrontar el estrés, porque con ello, se asume una posición positiva para enfrentar la adversidad como indica Navarro (2009), los estudiantes con una autoconfianza tienen enorme potencial en todo sentido Ralph (2014) y con su claridad emocional asumen las condiciones adversas como problemas directos a afrontar y resolver como refiere Puigbo et al. (2019) y Acevedo (2015). Los estudiantes presentaron la relación inversa en analogía a la confianza en sí mismos, con la cualidad de ser conscientes del momento presente, en el estudio de Baena et al. (2021), esta cualidad evidencia una reducción del estrés en estudiantes de primaria y secundaria. El grupo piloto, en el cual se encuentran estos estudiantes, es un factor importante, ya que tienen el apoyo familiar, las condiciones materiales y afectivas favorables del hogar y sobre todo la disposición personal de concretar metas académicas de excelencia, como característica de un grupo escolar selecto, así referido por Murillo y Martines-Garrido (2018). Estas condiciones, hacen posible una autoconfianza consciente que les permite afrontar con éxito el estrés académico, como menciona Villarejo et al. (2020), con algunas similitudes al grupo de estudiantes de los colegios de alto rendimiento COAR, como expone Alcazar y Balarin (2021).

Con el objetivo de relacionar la dimensión de la resiliencia: "sentirse bien solo" y el "estrés en el contexto del COVID-19", se reporta que fue una correlación cercana a una correlaciona negativa considerable ( $\mathrm{r}=-.717$ y p <0.01), en la percepción de un nivel alto de resiliencia en la dimensión "sentirse bien solo", sobre el estrés académico en su nivel leve y moderado con (61.54\% y 19.23\%) concordantemente, como se observa en la figura 3. Se concluye que existe evidencia estadística, que los estudiantes encuestados tiene un menor rango de estrés académico, por tener un alto rango de resiliencia en su dimensión sentirse bien solo; este resultado es análogo al reportado Salvatierra (2019), quien reportó una correlación superior a una negativa débil $(\mathrm{r}=-.27$ y $\mathrm{p}<0.05)$ para el aspecto: sentirse bien solo de la resiliencia y el estrés académico, con el estudio de García et al. (2021), en su estudio con estudiantes jóvenes indica que, la dimensión "aceptación de sí mismo" que sería paralela a "sentirse bien solo" (rho=-0,387 y $\mathrm{p}=<0.001$ ), significando que, cuanto mayor es la satisfacción de sentirse bien solo se tiene menor nivel de estrés académico. 
La relación reportada en el estudio, puede tener fundamento en la naturaleza de los estudiantes que pertenecen a un grupo de alto rendimiento académico, que institucionalmente la denominan "aula piloto" Treviño et al. (2016), aunque esta segregación no debería existir, ya que el sistema educativo peruano no lo considera, es una práctica común ejecutarla para poder tener cuadros representativos institucionales; así como satisfacer necesidades de aprendizaje y atención, lo cual si prevé el (CNEB, 2017). La relación considerable de menor estrés a favor de mayor sensación de sentirse bien solo, puede ser explicado desde la propia competencia: gestiona su aprendizaje de manera autónoma, transversal del CNEB (2017), con sus capacidades de definir metas, organizar estrategias para lograrla, y ajustar su desempeño para su logro, son definitivamente actitudes y aptitudes muy favorables para enfrentar el estrés académico y que se ven en el grupo estudiado, al pertenecer a aulas piloto que se diferencian de las demás, porque en ellas se tiene mejores condiciones materiales y no materiales para un trabajo, como refiere Murillo y Martinez-Garrido (2018); otro argumento explicativo es el apoyo de la familia a los estudiantes en su aislamiento, que sin poder estar con sus compañeros enfrentaron positivamente el estrés académico de las clases virtuales y su nueva exigencia (Caldera et al., 2017; Duque y Arias, 2021).

Por la naturaleza del estudio, no se exponen las correlaciones con otras dimensiones de la resiliencia, el estudio quedo ajustado a la aplicación de los instrumentos y la revisión de literatura específica, además, no fue posible aplicar otros instrumentos como la entrevista presencial por las condiciones de aislamiento a causa de la crisis sanitaria. Se podría considerar para posteriores estudios, la correlación con aulas no piloto y la comparación entre estas; así como: la diferencia de género, la dirección estratégica institucional, la mediación y acompańamiento de los docentes. Los hallazgos realizados son significativos por la utilidad que pueden darle a la organización al interior del colegio, como el departamento de tutoría, el concejo de docentes, la dirección de formación general y en el exterior, la organización de padres de familia y la comunidad.

\section{Conclusión}

A partir del trabajo realizado, se concluye que existe evidencia estadística que relaciona de modo inverso la resiliencia con sus dimensiones: "confianza en sí mismo" y "sentirse bien solo" con el estrés académico en el contexto COVID-19. Ello significa que a mayor nivel de resiliencia se tendrá un menor nivel de estrés; por eso, se debe involucrar, desde la familia y el colegio cualidades resilientes a los estudiantes, como la fuerza interior "confianza en sí mismo" y la independencia y autonomía como el "sentirse bien solo".

\section{Referencias}

Acevedo, H. (2015). La estimulación de las emociones y la confianza en si mismo como habilidades para la vida en estudiantes de la facultad de Educación de la Universidad de San Buenaventura Medellín [Tesis de maestría, Universidad de Manizales]. https://ridum.umanizales.edu.co/xmlui/handle/20.500.12746/2821

Alania, R., Llancari, R., de la Cruz, M., y Ortega, D. (2020). Adaptación del cuestionario del estrés académico SISCO SV al contexto de la crisis por el Covid-19. Socialium Revista de Ciencias Sociales, 4(12), 111-130. https://doi. org/10.26490/uncp.sl.2020.4.2.669

Alcazar, L., y Balarin, M. (2021). Evaluación del diseño e implementación de los colegios de alto rendimiento COAR: informe final. Ministerio de Educación del Perú. http://repositorio.minedu.gob.pe/handle/20.500.12799/7059

Anjicama, L. (2017). Propiedades psicométricas del inventario SISCO del estrés académico en universitarios de la ciudad de Trujillo [Tesis de Licenciatura, Universidad César Vallejo]. https://hdl.handle.net/20.500.12692/650

Asenjo, J., Linares, O., y Díaz, Y. (2021). Nivel de estrés académico en estudiantes de enfermería durante la pandemia de COVID-19. Revista Peruana de Investigación en Salud, 5(2), 59-66. https://doi.org/10.35839/repis.5.2.867

Baena, A., Ortiz, M., Marfil, M., y Granero, A. (2021). Mejora de los niveles de atención y estrés en los estudiantes a través de un programa de intervención Mindfulness. Revista de Psicodidáctica, 26(2), 132-142. https://doi. org/10.1016/j.psicod.2020.12.002 
Barraza, A. (2008). El estrés académico en alumnos de maestría y sus variables moduladoras: un diseño de diferencia de grupos. Avances en psicología latinoamericana, 26(2), 34-45. https://www.redalyc.org/pdf/799/79926212.pdf

Barraza, A., y Oscar, M. (2010). Estrés de examen, variables moduladoras y reprobación escolar. Revista Internacional de Psicología, 11(2), 1-18. https://doi.org/10.33670/18181023.v11i02.61

Barraza, A., y Silerio, J. (2007). El estrés académico en alumnos de educación media superior: un estudio comparativo. Revista Investigación Educativa, (7) 48-65. https://www.researchgate.net/publication/28175059_El_estres_ academico_en_los_alumnos_de_educacion_media_superior_Un_estudio_comparativo

Becońa, E. (2006). Resiliencia: definición, características y utilidad del concepto. Revista de Psicopatología y Psicología Clínica, 11(3), 102-112. https://doi.org/10.5944/rppc.vol.11.num.3.2006.4024

Berzosa, R. (2016). Estrés académico, engagement y resiliencia en estudiantes de enfermería [Trabajo de fin de grado, Universidad de Valladolid]. https://uvadoc.uva.es/bitstream/handle/10324/24508/TFG-H911. pdf?sequence $=18$ is Allowed $=y$

Cabrera, J., Cale, J., y Cabrera, E. (2019). La inclusión en el aula en escuelas con alto rendimiento escolar: Estudio de caso en escuelas de la provincia de Carchi, Ecuador. Espacios, 40(44), 3-12. https://www.revistaespacios.com/ a19v40n44/a19v40n44p03.pdf

Cahuana, F., y Carazas, D. R. (2018). Clima social familiar y resiliencia en adolescentes de 15 a 19 años de edad, internos del centro Juvenil de Diagnóstico y rehabilitación de Lima [Tesis de Licenciatura, Universidad Peruana Unión]. https://repositorio.upeu.edu.pe/bitstream/handle/20.500.12840/1009/Flor_Tesis_2018.pdf?sequence=5

Caldera, J. F., Pulido, B. E., y Martínez, M. G. (2007). Niveles del estrés y rendimiento académico de estudiantes de la carrera de Psicología del centro Universitario de Los Altos. Revista de Educación y Desarrollo, 77-82. https:// www.cucs.udg.mx/revistas/edu_desarrollo/anteriores/7/007_Caldera.pdf

Caldera, J., Reynoso, O., Mora, O., y Anaya, B. (2017). Modelo explicativo y predictivo de respuestas de estrés académico en bachilleres. Ansiedad y Estres, 23(1), 20-26. https://doi.org/10.1016/j.anyes.2017.02.002

López., I., y Calvete, E. (2016). Desarrollo del inventario de factores de resiliencia ante la adversidad. Ansiedad y Estrés, 2(2-3), 110-117. http://dx.doi.org/10.1016/j.anyes.2016.10.003

Canazas, B., y Díaz, H., y Cáceres, G. (2019). Resiliencia y apoyo social percibido en estudiantes de cuarto y quinto de secundaria del distrito de Ayaviri, 10(1), 23-37. https://doi.org/10.36901/psicologia.v10i1.748

Cañete, D., Cáceres, E., Soto, R., y Gómez, M. (2021). Educación a distancia en tiempo de pandemia en Paraguay. EDUTEC Revista Electrónica de Tecnología Educativa, (76), 181-196. https://doi.org/10.21556/ edutec.2021.76.1889

Caqueo, A., Urzúa, A., Aragón, D., Charles, C., El-Khatib, Z., Otu, A, \& Yaya, S. (2020). Mental Health and the COVID-19 Pandemic in Chile [Salud y la pandemia de COVID-19 en Chile]. . Psychological Trauma: Theory, Research, Practice, and Policy, 12(5), 521-524. http://dx.doi.org/10.1037/tra0000753

Castagnola, C., Cotrina, C., y Aguinaga, D. (2021). La resiliencia como factor fundamental en tiempos de Covid-19. Propósitos y Representaciones, 9(1), 10-20. http://dx.doi.org/1020511/pyr2021

Currículo Nacional de Educación Básica. (2017). Currículo Nacional de Educación Básica.1 Ministerio de Educación del Perú.

Correa, E., y Mendoza, J. (2020). Resiliencia según sexo en estudiantes de dos universidades de la ciudad de Cajamarca [Tesis de licenciatura, Universidad Privada Antonio Guillermo Urrelo]. http://repositorio.upagu.edu.pe/bitstream/ handle/UPAGU/1486/INFORME\%20DE\%20TESIS\%202021.pdf?sequence=1\&isAllowed=y

Diaz, D. L. (2011). Estrés laboral y sus factores de riesgo psicosocial. Revista CES Salud Pública, 2(1), 80-84. https:// www.researchgate.net/publication/277274612_Estres_laboral_y_sus_factores_de_riesgo_psicosocial

Duque, C., y Arias, M. (2021). La familia en la unidad de cuidados intensivos frente a una crisis situacional. Enfermería Intensiva, 112-118. https://doi.org/10.1016/j.enfi.2021.02.003 
García, K., Salazar, E., y Barja, J. (2021). Resiliencia y estrés percibido en estudiantes de Obstetricia de una universidad pública de Lima, Perú. FEM Revista de la Fundacion Educación Médica, 24(2), 95-99. https://dx.doi.org/10.33588/ fem.242.1117

Gonzáles, E. (2019). Nivel de resiliencia en adolescentes en una institucion educativa de Monsefu, Chiclayo, 2019. [Tesis de Licenciatura, Universidad Norbert Wiener]. http://repositorio.uwiener.edu.pe/xmlui/bitstream/ handle/123456789/3171/TESIS\%20Gonzales\%20Elva.pdf?sequence=1\&isAllowed=y

Gonzáles, L. (2020). Estrés académico en estudiantes universitarios asociado a la pandemia por Covid-19. Revista digital de la Universidad Autónoma de Chiapas, 9(25), 10-20. http://dx.doi.org/10.31644/IMASD.25.2020.a10

Hernández, B., y Velasco, H. (2000). Encuestas transversales. Salud Pública de México, 42(5), 447-455. https://www. scielosp.org/pdf/spm/2000.v42n5/447-455/es

Hernández, R., Fernández, C., y Baptista, P. (2014). Metodología de la Investigación. Mc Graw Hill, Educations.

Huarcaya, J. (2020). Consideraciones sobre la salud mental en la pandemia de COVID-19. Revista Peruana de Medicina Experimental y Salud Pública, 37(2), 327-334. https://doi.org/10.17843/rpmesp.2020.372.5419

Imbert, N. (2020). Covid 19-10 propuestas para pueblos y territorios más resilientes. Actualidad Jurídica Ambiental, 1-6. https:/www.actualidadjuridicaambiental.com/wp-content/uploads/2020/04/2020_04_06_Imbert_GreenCross-Covid.pdf

Jacobo, D. (2020). Reflexiones sobre la plataforma Aprendo en casa del Ministerio de educacion del Perú durante la pandemia Covid-19. Educación y pandemia. Una visión desde la Universidad, 35-44. http://catedraunesco.usmp. edu.pe/pdf/educacion-pandemia.pdf\#page $=35$

Kang, L., Li, Y., y Hu, S. (2020). The mental health of medicfal workers in Wuhan, China dealing with the 2019 novel coronavirus [La salud mental de los trabajadores médicos en Wuhan, China, lidiando con el nuevo coronavirus de 2019]. Lancent Pcychiatry, 7(3), https://doi.org/10.1016/S2215-0366(20)30047-X

Kim, J. (2019). Resilience and creativy in elementary scholl children [Resiliencia y creatividad en niños de primaria]. Revista de Investigación en Psicología, 22(1), 67-78. http://dx.doi.org/10.15381/rinvp.v22i1.16582

Kotliarenco, M., Cáceres, I., y Fontecilla, M. (1997). Estado del arte en resiliencia. Fundación W.K.Kellogg. http:// www.ugr.es/-javera/pdf/2-3-resiliencia\%20libro.pdf

León, A., Gonzáles, S., Gonzáles, N., y Barcelata, B. (2019). Estrés, autoeficacia, rendimiento académico y resiliencia en adultos emergentes. Electronic Journal of Research, 17(1), 129-148. https://doi.org/10.25115/ejrep.v17i47.2226

León, E. (2020). Evaluación del sistema, alfabetizacion inicial y “Aprendo en casa”. Educación, 1-9. https:// www.educaccionperu.org/wp-content/uploads/wp-post-to-pdf-enhanced-cache/1/evaluacion-de-sistemaalfabetizacion-inicial-y-aprendo-en-casa.pdf

Linares, C. E. (2021). Estrés académico en escolares de quinto de secundaria de dos instituciones educativas públicas en periodo de pandemia, Lima 2020. [Tesis de Maestría, Universidad César Vallejo]. https://repositorio.ucv.edu.pe/ handle/20.500.12692/59967

López., y Calvete, E. (2016). Desarrollo del inventario de factores de resiliencia ante la adversidad. Ansiedad y Estrés, 2(2-3), 110-117. http://dx.doi.org/10.1016/j.anyes.2016.10.003

Lozano, A. (2020). Impacto de la epidemia del Coronavirus (COVID-19) en la salud mental del personal de salud y en la población general de China. Neuro Psiquiatr, 83(1), 50-62. http://www.scielo.org.pe/scielo.php?pid=S003485972020000100051\&script=sci_abstract

Luthar, S., Ciccheti, D., y Becker, B. (2000). The construct of resilience: A critical evaluation and guidelines for future work [El constructo de resiliencia: una evaluación crítica y pautas para el trabajo futuro]. Child development, 71(3), 543-562. https:/www.ncbi.nlm.nih.gov/pmc/articles/PMC1885202/

Maldonado, E., y Paucar, E. (2021). Estrés académico y resiliencia en estudiantes de la facultad de educación de una universidad estatal de Huancayo-2020 [Tesis de Licenciatura, Universidad Peruana Los Andes]. http://www. repositorio.upla.edu.pe/handle/20.500.12848/2222 
Mamani, G. (2020). Aprendo en casa. Lecciones en el contexto de la emergencia sanitaria por la COVID-19. América Critica, 4(2),181-183. https://doi.org/10.13125/americacritica/4349

Martínez, R., Tuya, L., Pérez, A., y Cánovas, A. (2009). El coeficiente de correlación de los rangos de Spearman caracterizacion. Revista haban cienc med La Habana, 3(2), 1-19. http://scielo.sld.cu/pdf/rhcm/v8n2/ rhcm17209.pdf

Melgosa, J. (1995). Nuevo estilo de vida sin estrés. Safeliz Madrid.

Méndez, A. (2020). Resultados académicos, cognitivos y físicos de dos estrategias para integrar movimientos en el aula: clases activas y descansos activos. SPORT TK Revista Euroamericana de Ciencias del Deporte, 9(1), 63-74. https:// dialnet.unirioja.es/servlet/articulo?codigo $=7245172$

Morales, J. (2017). Resiliencia y estrés académico en estudiantes de la facultad de ciencias de la salud de la Universidad de Ciencias y Humanidades [Tesis de Maestría, Universidad Nacional Enrique Guzman y Valle La Cantuta]. https:// repositorio.une.edu.pe/bitstream/handle/UNE/1854/TM\%20CE-Du\%203644\%20M1\%20-\%20Morales\%20 Martinez.pdf?sequence $=1 \&$ is Allowed $=y$

Moran, S. (2018). Resiliencia en adolescentes institucionalizado del centro de atencion residencial Hogar de San PedritoNuevo Chimbote 2017 [Tesis de Licenciatura, Universidad San Pedro]. http://repositorio.usanpedro.pe/bitstream/ handle/USANPEDRO/8991/Tesis_59437.pdf?sequence=18isAllowed=y

Muntané, J. (2010). Introducción a la investigación básica. RAPD Online, 32(3), 221-227. https://www. researchgate.net/profile/Jordi-Muntane/publication/341343398_Introduccion_a_la_Investigacion_basica/ links/5ebb9e7d92851c11a8650cf9/Introduccion-a-la-Investigacion-basica.pdf

Murillo, F., y Martínes-Garrido, C. (2018). Factores de aula asociados al desarrollo integral de los estudiantes: Un estudio observacional. Estudios Pedagógicos, 44(1), 181-205. http://dx.doi.org/10.4067/S0718-07052018000100181

Naranjo, J. (2009). Una revisión teórica sobre estrés y algunos aspectos relevantes de este en el ámbito educativo. Revista Educación, 33(2), 171-190. https://www.redalyc.org/pdf/440/44012058011.pdf

Navarro, M. (2009). Autoconocimiento y autoestima. Temas para Educación, (5),1-9. https://www.feandalucia.ccoo.es/ docu/p5sd6409.pdf

Novella, A. (2002). Incremento de la resiliencia luego de la aplicacionde un programa de psicoterapia breve en madres adolescentes. [Tesis de Maestría, Universidad Nacional Mayor de San Marcos]. https://dialnet.unirioja.es/ descarga/articulo/3052961.pdf

Nunja, M. (2016). Propiedades psicométricas de la escala de resiliencia en estudiantes de institutos técnicos superiores de la ciudad de Trujillo. [Tesis de Licenciatura, Universidad César Vallejo]. https://repositorio.ucv.edu.pe/bitstream/ handle/20.500.12692/322/nunja_am.pdf?sequence=1\&isAllowed=y

Oltra, C., y Boso, A. (2020). Lecciones aprendidas de la crisis del coronavirus: preparación y Resiliencia social. Revista Española de Sociologia, 29(3), 769-775. https://dialnet.unirioja.es/servlet/articulo?codigo=7540815

Palomino, C., y Huarcaya, J. (2020). Transtornos por estrés debido a la cuarentena durante la pandemia por la COVID-19. Horizonte Médico ISSN-2227-3530, 20(4), 121-129. https://doi.org/10.24265/horizmed.2020. v20n 4.10

Pérez, J., Dorado, A., Rodríguez, M., y López, J. (2020). Resiliencia para promoción de la salud en la crisis Covid-19 en España. Revista de Ciencias Sociales, 26(4), 52-63. https://dialnet.unirioja.es/servlet/articulo?codigo=7687026

Pérez, M., Álvarez, N., y Rodríguez, A. (2020). Repercusión psicológica y social de la pandemia COVID-19. Revista Electrónica Medimay, 27(2), 252-261. https://www.medigraphic.com/pdfs/revciemedhab/cmh-2020/ cmh202p.pdf

Prado, R., y del Aguila, M. (2003). Diferencia en la resiliencia segun género y nivel socioeconómico en adolescentes. Persona, 179-196. https://www.redalyc.org/pdf/1471/147118110009.pdf

Puigbo, J., Edo, S., Rovira, T.,Limonero, J. J. (2019). Influencia de la inteligencia emocional percibida en el afrontamiento del estrés. Ansiedad y Estrés, 25(1)1-6. https://doi.org/10.1016/j.anyes.2019.01.003. 
Ralph, E. (2014). La confianza en si mismo. FV Editions.

Raymundo, Y. (2021). Estrés laboral y salud mental en el contexto de lapandemia en docentes de educación secundaria Huancavelica-2020. [Tesis de Licenciatura, Universidad Peruana de los Andes]. https://repositorio.upla.edu.pe/ bitstream/handle/20.500.12848/2275/TESIS\%20FINAL.pdf?sequence=1\&isAllowed=y

Reyes, P., Resendiz, R., Alcazar, O., y Reidi, M. (2017). Las estrategias de afrontamiento que utilizan los adolescentes ante situaciones que provocan miedo. Psicogente, 20(38), 240-255. http://www.scielo.org.co/pdf/psico/ v20n38/0124-0137-psico-20-38-00240.pdf

Rodriguez, C. (2021). Cinco retos psicológicos de la crisis del COVID-19. Journal of Negative and No Positive Results, 5(6), 583-588. https://doi.org/10.19230/jonnpr.3662

Rojas, Y. (2016). Resiliencia y afrontamiento del estrés en estudiantes de una institución secundaria de Chiclayo. [Tesis de Licenciatura, Universidad Privada Seńor de Sipán]. https://repositorio.uss.edu.pe/bitstream/ handle/20.500.12802/3241/ROJAS_CASTRO_YASARI.pdf?sequence=7\&isAllowed=y

Román, C., y Hernández, Y. (2011). El estrés académico: Una revisión crítica del concepto desde las ciencias de la educación. Revista Electrónica de Psicología Iztacala, 14(2), 1-14. https://www.iztacala.unam.mx/carreras/ psicologia/psiclin/vol14num2/Vol14No2Art1.pdf

Roman, F., Forés, A., Calandri, I., Gautreaux, R., Antúnez, A., Ordhei, D., Calle, L., Poenitz, V., Correa, K.L., Torresi, S., Barcelo, E., Conejo, M.,Ponnet, V., y Allegri, R. (2020). Resiliencia de docentes en distanciamiento social preventivo obligatorio durante la pandemia COVID-19. Journal of Neuroeducation, 1(1), 76-87. https://revistes. ub.edu/index.php/joned/article/view/31727

Salvatierra, L. (2019). Resiliencia y estrés académico en estudiantes de un Conservatorio de Música Público de Trujillo. [Tesis de Maestría, Universidad Cesar Vallejo]. https://repositorio.ucv.edu.pe/bitstream/handle/20.500.12692/37985/ salvatierra_ml.pdf?sequence=2

Serrano, A., y Sanz, R. (2019). Reflexiones y propuestas prácticas para desarrollar la capacidad de resiliencia frente a los conflictos en la escuela. Publicaciones, 49(1), 177-190.

https://dialnet.unirioja.es/servlet/articulo?codigo=7039527

Solano, A. (2020). Resiliencia y Covid 19. Revista Colombiana de Obstetricia y Ginecología, 71(1), 1-5. https://doi. org/10.18597/issn.0034-7434

Tamayo, G. (2001). Diseños muestrales en la investigación. Semestre Académico, 4(7), 1-14. https://dialnet.unirioja.es/ servlet/articulo?codigo $=5262273$

Tomas, A., y Lescano, S. (2003). Situación de las habilidades sociales en escolares del Perú, implementación de la linea de base de habilidades sociales. Ministerio de Educación del Perú.

Treviño, E., Villalobos, C., Vielma, C., Hernández, C., y Valenzuela, J. P. (2016). Trayectorias escolares de los estudiantes y agrupamiento al interior del aula en los colegios chilenos de enseñanza media. Análisis de la heterogeneidad académica al interior de las escuelas. Pensamiento Educativo, Revista de Investigación Educacional Latinoamericana, 53(2), 1-17. https://revistaschilenas.uchile.cl/handle/2250/133067

Uriarte, J. (2005). La resiliencia. Una nueva perspectiva en psicología del desarrollo. Revista de Psicodidáctica, 10(2), 61-79. https://www.redalyc.org/pdf/175/17510206.pdf

Velavan, T., y Meyer, C. (2020). The COVID-19 epidemic. Trop Med Int Health, 25(3), 278-280. https://doi. org/10.1111/tmi.13383

Velezmoro, G. (2018). Cohesión familiar y resiliencia en adolescentes de una institución educativa estatal de Trujillo [Tesis de Maestría, Universidad Peruana Cayetano Heredia]. https://hdl.handle.net/20.500.12866/3594

Verastegui, A. (2020). Estrés académico y resiliencia en estudiantes de una universidad de Lima norte [Tesis de Licenciatura, Universidad Privada del Norte]. https://repositorio.upn.edu.pe/bitstream/handle/11537/24260/Verastegui\%20 Torrejon,\%20Maria\%20Alejandra.pdf?sequence=7 
Villarejo, S., Martinez, J., y Garcia, O. (2020). Estilos parentales y su contribución al ajuste personal y social de los hijos. Ansiedad y Estrés, 26(1), 1-8. https://doi.org/10.1016/j.anyes.2019.12.001

Vivanco, R.E., Pérez, R., y Alvarez, M. M. (2021). Diseño de un corto documental para evidenciar el estrés académico generado por las clases virtuales en los estudiantes del 5 to grado de secundaria en el contexto de la pandemia por la Covid-19 en una institucion educativa privada del distrito de Pachacamac [Tesis de Licenciatura. Universidad San Ignacio de Loyola]. http://repositorio.usil.edu.pe/handle/USIL/11526

Wagnild, G., y Young, H. (1993). Development and psychometric evaluation of resilience Scale [Escala de desarrollo y evaluación psicométrica de la resiliencia]. Journal of Nursing Measurement, 1(2), 165-177. https://pubmed.ncbi. nlm.nih.gov/7850498/

\section{Notas al final}

1 Profesora de educación secundaria especialidad Comunicación del Instituto Superior Pedagógico Gregoria Santos Sicuani- Perú, Bachiller en educación por la Universidad Nacional de San Antonio Abad del Cusco, segunda especialidad en Educación Técnico productiva por la Universidad José Carlos Mariátegui de Moquegua- Perú y con maestría en Psicología Educativa de la Universidad César Vallejo -Perú.

2 Licenciada en Educación en Computación e Informática de la Universidad Alas Peruanas, segunda especialidad en Educación Inglés de la Universidad José Carlos Mariátegui de Moquegua- Perú, egresada de la Maestría en Administración de la Educación de la Universidad César Vallejo- Perú, Capacitadora de Aula Digital de la Fundación Telefónica.

3 Profesor de educación secundaria especialidad Matemática del Instituto Superior Pedagógico Gregoria Santos Sicuani- Perú, Licenciado en Educación secundaria Matemática por la Universidad Nacional Mayor de San Marcos-Lima, segunda especialidad en Educación para el Trabajo y Formación Ocupacional por la Universidad Nacional de San Agustín de Arequipa-Perú, egresado de la Maestría en Investigación y Docencia en Educación Superior por la Universidad Néstor Cáceres Velásquez Juliaca-Perú.

4 Licenciada en Educación Primaria por la Universidad Nacional de San Antonio Abad del Cusco, segunda especialidad en Educación Intercultural Bilingüe por el Instituto Pedagógico Nacional de Monterrico-Perú, diplomado en Tutoría Educativa por la Universidad Santiago Antúnez de Mayolo -Perú, especialización en Dirección y Gerencia Educativa por la Universidad nacional de Educación Enrique Guzmán y Valle La Cantuta-Perú, egresada de la Maestría en Psicología Educativa de la Universidad Cesar Vallejo-Perú. 